\title{
SUPPLEMENT TO "RECONSTRUCTING PAST TEMPERATURES FROM NATURAL PROXIES AND ESTIMATED CLIMATE FORCINGS USING SHORT- AND LONG-MEMORY MODELS"
}

\author{
By Luis Barboza, Bo Li, Martin Tingley and Frederi Viens
}

\section{ONLINE SUPPLEMENT A}

A.1. Background on long memory models. As mentioned in the introduction, long-memory estimation is typically difficult both in theory and in practice; fundamental stochastic analysis research on this question is ongoing. In discrete time series, the long-memory autoregressive moving average (ARMA) and autoregressive conditional heteroskedasticity (ARCH) models that are popular in financial econometrics (see Granger and Joyeux (1980) and Baillie, Bollerslev and Mikkelsen (1996) for variations of ARMA and $\mathrm{ARCH}$ ), cannot be adapted to our Bayesian context. These models are complex and are not explicitly specified in terms of distributions; they often rely on long data series or high-frequency in-fill data. It can be argued that the only models for which the discrete-time parametric memory-length estimation is straightforward and reliable in finite samples are those that come from continuous-time underlying models which exhibit self-similarity (identical distribution at all time scales), of which the typical example is fractional Brownian motion (fBm). Pioneering work in this direction was performed in Coeurjolly (2001), and further details and developments were provided in Chronopoulou, Viens and Tudor (2009). Long-memory structures which are slightly more complex than those studied therein are already difficult to estimate.

For instance, the so-called fractional Ornstein-Uhlenbeck (fOU) process has a well-known estimator for its drift parameter in continuous time (Kleptsyna and Le Breton, 2002), and with discrete data for increasing horizon asymptotics (Tudor and Viens, 2007), but the question of estimating its long-memory parameter remains elusive. When in-fill asymptotics can be attained in the data this can be accomplished via path regularity in continuous time (see Istas and Lang (1997) and its application in Brouste and Iacus (2012)). Neither this, nor the use of increasing-horizon asymptotics in Tudor and Viens (2007) are applicable in our paleoclimatology situation, because our observation frequency is not high enough and our calibration period is too short. 
A.1.1. Properties of $f B m$ and $f G n$. Fractional Gaussian noise (fGn) is defined as the first-order increment sequence of $\mathrm{fBm}$. Information on $\mathrm{fBm}$ and fGn can be found in standard references; Biagini et al. (2010) provides an excellent mathematical treatment. The following is a short summary of properties relevant to our study. Fractional Brownian motion $(\mathrm{fBm}), B^{H}=$ $\left\{B_{t}^{H}: t \geq 0\right\}$ with Hurst parameter $H \in(0,1)$, is a continuous centered Gaussian process with covariance function:

$$
E\left[B_{t}^{H} B_{s}^{H}\right]=\frac{1}{2}\left(t^{2 H}+s^{2 H}-|t-s|^{2 H}\right)
$$

for $t, s>0$. From this definition we immediately deduce fundamental properties:

- $B_{0}^{H}=0$,

- $B^{H}$ has stationary increments:

$$
E\left[\left(B_{t+h}^{H}-B_{s+h}^{H}\right)^{2}\right]=E\left[\left(B_{t}^{H}-B_{s}^{H}\right)^{2}\right]=|t-s|^{2 H}
$$

but these increments are not independent (see long-range dependence property below), except if $H=1 / 2$, in which case $B_{t}^{1 / 2}$ is the standard Brownian motion.

- The increment process $N^{H}$ defined by $N_{t}^{H}:=B_{t+\Delta t}^{H}-B_{t}^{H}$ is called a fractional Gaussian noise (fGn), for fixed time step $\Delta t>0 . N^{H}$ is a stationary, mean-zero Gaussian process, with constant variance $(\Delta t)^{2 H}$ and autocovariance function given by:

$$
E\left[N_{s+t}^{H} N_{s}^{H}\right]=\frac{1}{2}\left[|t+\Delta t|^{2 H}+|t-\Delta t|^{2 H}-2|t|^{2 H}\right] .
$$

For convenience, $\Delta t$ can be interpreted as one unit of time, and $t$ as an integer. In our study, $t$ is measured in years.

We say that a stationary process $\left\{X_{t}, t \in \mathbb{N}\right\}$ is a "long-range dependent" process, or "long-memory" process, if its autocovariance function $\rho(t)=$ $E\left[X_{s+t} X_{s}\right]$ satisfies:

$$
\lim _{t \rightarrow \infty} \frac{\rho(t)}{t^{-M}}=c_{1}
$$

for some constant $c_{1}>0$ and $M \in(0,1)$, so that $\sum_{n=1}^{\infty} \rho(t)=\infty$. For a process such as $\mathrm{fBm}$, which is not stationary, but has stationary increments, we say that is has long-range dependence (long memory) if its increment process has long range dependence. There is an alternative condition for 
long-range dependence in terms of the spectral behavior of the stationary process $X_{n}$ :

$$
\lim _{\lambda \rightarrow 0} \frac{f(\lambda)}{|\lambda|^{-D} G(|\lambda|)}=c_{2}
$$

where $c_{2}>0, D \in(0,1)$ and $G$ is a slow varying function at 0 . It turns out that fGn (and thus fBm) have long-range dependence for $H>\frac{1}{2}$. Indeed, for these processes we have $M=2-2 H \in(0,1)$ and $D=2 H-1 \in$ $(0,1)$ in the above limits, with the constants $c_{1}=H(2 H-1)$ and $c_{2}=$ $\pi /(H \Gamma(2 H) \sin (\pi H))$. For further details, see Chapter 5 in Nualart (2010) and Chapter 7 in Samorodnitsky and Taqqu (1994).

\section{A.1.2. Hypothesis testing for possible long-memory.}

Robinson's test(Robinson, 1995) Define the semiparametric Gaussian estimate of $H$ as:

$$
\hat{H}=\operatorname{argmin}_{H}\left\{\log \left(\frac{1}{m} \sum_{j=1}^{m} \lambda_{j}^{2 H-1} I\left(\lambda_{j}\right)\right)-(2 H-1) \frac{1}{m} \sum_{j=1}^{m} \log \lambda_{j}\right\}
$$

where $\lambda_{j}=2 \pi j / n$ for $j=1, \ldots, m, m=\frac{n}{2}$, and $I(\cdot)$ is the Periodogram estimator constructed using the observations $X_{1}, X_{2}, \cdots, X_{n}$. Assume $X$ 's spectral density satisfies $f(\lambda) \sim G \lambda^{1-2 H}$ (for some fixed $H)$. Under rather weak distributional assumptions on $X$, Robinson (1995) proves that $\hat{H}$ is a consistent estimator of $H$, and is asymptotic normal in the sense that $2 m^{\frac{1}{2}}(\hat{H}-H)$ converges to a standard normal distribution as $m \rightarrow \infty$. This asymptotic behavior implies that we can test the following null and alternative hypotheses (Robinson's test):

Null Hypothesis: $H=0.5$ (no memory)

Alternative: $H>0.5$ (presence of memory)

Davies and Harte's test (Davies and Harte, 1987) Assume that $X=\left(X_{1}, \ldots, X_{n}\right)$ are normally distributed with a autocorrelation function given in (A.1). In order to test the same alternatives as Robinson's test (A.2), Davies and Harte derived a locally optimal test with critical region:

$$
X^{\prime}\left[c\left(I-11^{\prime} / n\right)-\left(I-11^{\prime} / n\right) T\left(I-11^{\prime} / n\right)\right] X<0
$$

where

$$
T_{i, j}= \begin{cases}0 & i=j, \\ \log 2 & i=j \pm 1 \\ \frac{1}{2}\left[|i-j| \log \left(1-(i-j)^{-2}\right)+\log \frac{|i-j|+1}{|i-j|-1}\right] & \text { otherwise }\end{cases}
$$


and $c$ is a constant chosen to attain a desired significance level in the test.

Goodness of fit test (Beran, 1992) Let $X_{t}$ be a stationary Gaussian process with spectral density $f(\lambda)$ satisfying $f(\lambda) \sim G \lambda^{1-2 H}$ (assume $H$ is known). Let $X_{1}, \ldots, X_{n}$ be the $n$ observatiouns of $X$. If $f(\lambda, H)$ is the spectral density of the fGn with Hurst parameter $H$, then we want to test, for all $\lambda$, the Null Hypothesis $f(\lambda)=f(\lambda, H)$ against the Alternative: $f(\lambda) \neq f(\lambda, H)$. If we take $\hat{H}$ as the Whittle estimator of $H$ (see Beran, 1994), then Beran proposes the statistic:

$$
T_{n}(\hat{H})=\frac{A_{n}(\hat{H})}{B_{n}^{2}(\hat{H})}
$$

with $A_{n}(\hat{H})=\frac{4 \pi}{n} \sum_{k=1}^{n^{*}}\left[\frac{I\left(s_{j}\right)}{f\left(s_{j}, \hat{H}\right)}\right]^{2}$ and $B_{n}(\hat{H})=\frac{4 \pi}{n} \sum_{k=1}^{n^{*}} \frac{I\left(s_{j}\right)}{f\left(s_{j}, \hat{H}\right)}$, where $s_{j}=2 \pi j / n, I(\cdot)$ is the periodogram as in Robinson's test, and $n^{*}$ is the integer part of $\frac{1}{2}(n-1)$. Under mild technical assumptions on the distribution of $X$ 's, the Null distribution $T_{n}$ is asymptotically normal with mean $\pi^{-1}$ and variance $2 \pi^{-2} n^{-1}$, which results in Beran's test.

A.2. Multitaper Estimator. The general theory of spectral estimation, including the multitaper estimator, can be found in Thomson (1982) and Percival and Walden (1993) among others. Here we summarize relevant results concerning multitaper estimation. Suppose we have observations $X_{1}, X_{2}, \ldots, X_{N}$ of a stationary process $\left\{X_{t}\right\}$ with mean 0 , variance $\sigma^{2}$ and spectral density function $S(\cdot)$. The observations are separated in time by $\Delta t$. The direct spectral estimator is defined as:

$$
\hat{S}^{(d)}(f)=\Delta t\left|\sum_{t=1}^{N} h_{t} X_{t} e^{-i 2 \pi f t \Delta t}\right|^{2}
$$

where $\left\{h_{t}\right\}$ is a sequence of real-valued constants (tapers) and $f \in\left[-\frac{1}{2 \Delta t}, \frac{1}{2 \Delta t}\right]$. The Fourier transform of $\left\{h_{t}\right\}$ will be denoted as $H(f)$. It is not hard to conclude the following (defining $f_{N}=\frac{1}{2 \Delta t}$ ):

$$
E\left[\hat{S}^{(d)}(f)\right]=\int_{-\frac{1}{2 \Delta t}}^{\frac{1}{2 \Delta t}} \mathcal{H}\left(f-f^{\prime}\right) S\left(f^{\prime}\right) d f^{\prime}
$$


where $\mathcal{H}(f)=\frac{|H(f)|^{2}}{\Delta t}$. It we ask $h_{t}$ to be normalized $\left(\sum_{t=1}^{N} h_{t}^{2}=1\right)$, then in the case of a white noise with variance $\sigma^{2}$ and $S(t)=\sigma^{2} \Delta t$ we have:

$$
E\left[\hat{S}^{(d)}(f)\right]=\sigma^{2} \Delta t \int_{-f_{N}}^{f_{N}} \mathcal{H}(f) d f=\sigma^{2} \Delta t=S(t) .
$$

Hence the direct spectral estimator is unbiased. Moreover, in the case of $\left\{h_{t}=\frac{1}{\sqrt{N}}\right\}$, we have the usual Periodogram estimator with:

$$
\begin{aligned}
\mathcal{H}(f) & =N \Delta t \mathcal{D}_{N}^{2}(f \Delta t) \\
& =\frac{\Delta t \sin ^{2}(N \pi f \Delta t)}{N \sin ^{2}(\pi f \Delta t)}
\end{aligned}
$$

where $\mathcal{D}_{N}(\cdot)$ is the Dirichlet's kernel. In this case $\mathcal{H}(f)$ is usually denoted as $\mathcal{F}(f) . \mathcal{F}(f)$ has interesting properties, one of them is that it acts like a Dirac delta function with an infinite spike at $f=0$ when $N \rightarrow \infty$. This implies:

$$
\lim _{N \rightarrow \infty} E\left[\hat{S}^{(p)}(f)\right]=S(f) .
$$

One of the main problems of the periodogram is that series with large spectral range ${ }^{1}$ have a large bias due to the existence of significant sidelobes on $\mathcal{F}(f)$. This kind of bias is known as spectral leakage. The main idea of tapering is to provide smaller sidelobes in $\mathcal{H}$ than $\mathcal{F}$ has. A possible way to solve this problem is by means of the Slepian tapers. The main idea is to solve the concentration problem:

$$
\max _{\left\{h_{t}\right\}} \beta^{2}(W)
$$

where $W>0$ is fixed, and assuming $\Delta t=1$,

$$
\beta^{2}(W)=\int_{W}^{W}|\mathcal{H}(f)|^{2} d f / \int_{-1 / 2}^{1 / 2}|\mathcal{H}(f)|^{2} d f .
$$

This problem leads to the solution:

$$
h_{t, 0}=\frac{1}{\lambda_{0}(N, W)} \int_{-W}^{W} U_{0}(f, N, W) e^{i 2 \pi f\left[t-\frac{N-1}{2}\right]} d f
$$

\footnotetext{
${ }^{1}$ the spectral range can be defined as $\log \frac{\max S(f)}{\min S(f)}$
} 
for $t=0, \pm 1, \ldots$; where $U_{0}(\cdot, N, W)$ and $\lambda_{0}(\cdot, N, W)$ are the first eigenvector and eigenvalue of the system:

$$
\int_{-W}^{W} N \mathcal{D}_{N}\left(f^{\prime}-f\right) U(f) d f=\lambda U(f)
$$

In general $U_{k}(\cdot, N, W)$ is called the $\mathrm{k}$-th order discrete prolate spheroidal wave function and $h_{k}$ is the Slepian sequence of $\mathrm{k}$-th order:

$$
h_{t, k}=\frac{(-1)^{k}}{\epsilon_{k} \lambda_{k}(N, W)} \int U_{k}(f, N, W) e^{i 2 \pi f\left[t-\frac{N-1}{2}\right]} d f
$$

We then define the multitaper spectral estimator by:

$$
\hat{S}^{(m t)}(f)=\frac{1}{K} \sum_{k=0}^{K-1} \hat{S}_{k}^{(m t)}(f)
$$

where

$$
\hat{S}_{k}^{(m t)}(f)=\Delta t\left|\sum_{t=1}^{N} h_{t, k} X_{t} e^{-i 2 \pi f t \Delta t}\right|^{2}
$$

The multi taper estimator can be improved by considering a set of weights $b_{k}(f)$ such that the spectral leakage is minimized. It is possible to define such weights by considering an approximation of the Fourier transform of the time series; see Thomson (1982) and Percival and Walden (1993) for further details. The weights are then computed using an iterative procedure based on minimization of the spectral leakage. The algorithm generally converges after a few iterations (see Percival and Walden, 1993) and finally it is possible to define the Adaptive Multitaper spectral estimator as:

$$
S^{(a m t)}(f)=\frac{\sum_{k=0}^{K-1} b_{k}^{2}(f) \lambda_{k} \hat{S}_{k}^{m t}(f)}{\sum_{k=0}^{K-1} b_{k}^{2}(f) \lambda_{k}}
$$

In order to calculate the multitaper estimators in Figure 2 and 3 in the main document, we employed an estimator with $K=10$ (number of tapers) and $N W=5$. Hence, the design bandwidth (see Walden, McCoy and Percival (1995)) is approximately $2 \times 0.0602=0.1204$, which is a good approximation of the effective bandwidth of the multitaper estimation in the long-memory case. 
A.3. Scoring Rules. Gneiting and Raftery (2007) define scoring rules as "summary measures for the evaluation of probability forecasts, by assigning a numerical score based on the predictive distribution and on the event or value that materializes". Scoring rules are designed to reward both calibration quality and the sharpness of predictive distributions. Sharpness is understood as the concentration of the predictive distribution and is related to forecast precision (see Gneiting, Balabdaoui and Raftery (2007)).

"Proper scoring rules" are those with expected value that is minimized when the predictive distribution is equal to the true distribution, and are considered optimal (for more technical details, see Gneiting and Raftery, 2007). There are several examples of proper rules, and they depend on the type of data analyzed: for categorical data, the Brier, Spherical and Logarithmic scores; for continuous variables, the Continuous Ranked Probability score and Energy score; and for quantiles, the Interval score. See (see Gneiting and Raftery, 2007) for details and additional proper scoring rules.

In the current analysis we obtain samples from the posterior distribution of the temperature anomalies, and consider two proper scoring rules to assess these predictive distributions.

A.3.1. Continuous Ranked Probability score (CRPS). This score is defined in terms of the predictive distribution function $(F)$ directly (see Gneiting, Balabdaoui and Raftery, 2007):

$$
C R P S(F, x)=\int_{-\infty}^{\infty}\{F(y)-\mathbf{1}(y \geq x)\}^{2} d y
$$

where $x$ is the observed realization of the true distribution. Gneiting and Raftery (2007) give an alternative representation as:

$$
\operatorname{CRPS}(F, x)=E_{F}|X-x|-\frac{1}{2} E_{F}\left|X-X^{\prime}\right|
$$

where $E_{F}$ is the expectation with respect to the predictive distribution $F$ and $X^{\prime}$ is an independent copy of $X$ with the same law $F$. The main advantage of this representation is that under $R$ MCMC samples of the predictive $F$ $\left(y_{1}, \ldots, y_{R}\right)$ and an independent set of $R$ MCMC samples from the same distribution $\left(\tilde{y}_{1}, \ldots, \tilde{y}_{R}\right)$, we can approximate the $C R P S$ as (Gschlößl and Czado (2007)):

$$
\frac{1}{R} \sum_{i=1}^{R}\left[\left|y_{i}-x\right|-\frac{\left|y_{i}-\tilde{y}_{i}\right|}{2}\right] .
$$


If the realization $x_{t}$ depends on time $t$ (as well as the predictive distribution $F_{t}$ ), we can compute the mean CRPS score as:

$$
C R P S=\frac{1}{M} \sum_{t=1}^{M} C R P S\left(F_{t}, x_{t}\right)
$$

A.3.2. Interval score. The interval score is a special case of the quantile score (See equation 42 in Gneiting and Raftery, 2007). These scores are useful when we are able to compute exact or approximate quantiles of the predictive distribution $F$. This is the main reason why we were able to calculate this score under the normality assumption of Mann et al. (2008), as well as under our MCMC posterior calculations for comparison purposes. In the MCMC case, we compute the empirical $\alpha / 2$ and $1-\alpha / 2$ quantiles of the posterior distribution $F_{t}$, let us call them $l_{t}$ and $u_{t}$ respectively. Hence the interval score at level $1-\alpha$ is defined as (Gschlößl and Czado (2007)):

$$
I S_{(1-\alpha) \%}\left(l_{t}, u_{t}, x_{t}\right)= \begin{cases}2 \alpha\left(u_{t}-l_{t}\right)+4\left(l_{t}-x_{t}\right) & \text { if } x_{t} \leq l_{t} \\ 2 \alpha\left(u_{t}-l_{t}\right) & \text { if } l_{t} \leq x_{t} \leq u_{t} \\ 2 \alpha\left(u_{t}-l_{t}\right)+4\left(x_{t}-u_{t}\right) & \text { if } x_{t} \geq u_{t}\end{cases}
$$

and we can compute the mean IS at level $1-\alpha$ using the same formula as (A.3).

A.4. MCMC Posterior Distributions. In what follows, the notation $[X \mid Y]$ indicates the conditional probability of the random variable $X$ given $Y$. Suppose we have the following prior distributions:

- $\alpha \sim N_{2}\left(\hat{\alpha}, \hat{D}_{\alpha}\right)$

- $\beta \sim N_{4}\left(\hat{\beta}, \hat{D}_{\beta}\right)$

- $\sigma_{P}^{2} \sim I G\left(q_{1}, r_{1}\right)$

- $\sigma_{T}^{2} \sim I G\left(q_{2}, r_{2}\right)$

- $H \sim \operatorname{Unif}(0,1)$

- $K \sim \operatorname{Unif}(0,1)$

where all the prior parameters are treated as known constants. For the temperature reconstructions in this work, we set $\hat{\alpha}=(0,1), \hat{\beta}=(0,1,1,1)$, $\hat{D}_{\alpha}=I_{2}, \hat{D}_{\beta}=I_{4}, q_{1}=q_{2}=2, r_{1}=r_{2}=0.1$.

We first write the model of Eq. (3.4) in matrix form:

$$
\left\{\begin{array}{l}
\mathbf{R P}=\alpha_{0}+\alpha_{1} \mathbf{T}+\sigma_{P} \eta(H)=\tilde{\mathbf{T}} \alpha+\sigma_{P} \eta(H) \\
\mathbf{T}=\beta_{0}+\beta_{1} \mathbf{S}+\beta_{2} \tilde{\mathbf{V}}+\beta_{3} \tilde{\mathbf{C}}+\sigma_{T} \epsilon(K)=\mathbf{F} \beta+\sigma_{T} \epsilon(K)
\end{array}\right.
$$


for vectors $\mathbf{R P}$ and $\mathbf{T}$ of size $M \times 1$. Also assume that $\mathbf{T}=\left[\mathbf{T}_{1}, \mathbf{T}_{2}\right]$ where $\mathbf{T}_{1}$ is a vector of size $M-L \times 1$ and $\mathbf{T}_{2}$ has size $L \times 1$ where $L<M$. Let $\Sigma_{H}$ and $\Sigma_{K}$ be the covariance matrices of $\eta(H)$ and $\epsilon(K)$ respectively.

After some calculations we can deduce the following posterior distributions:

Distribution of $\mathbf{T} \mid \mathbf{R P}, \theta$.

$\begin{aligned} {[\mathbf{T} \mid \mathbf{R P}, \theta] } & \propto \exp \left[-\frac{1}{2}\left[\mathbf{T}^{T}\left(\frac{\alpha_{1}^{2}}{\sigma_{P}^{2}} \Sigma_{H}^{-1}+\frac{\Sigma_{K}^{-1}}{\sigma_{T}^{2}}\right) \mathbf{T}-2\left(\frac{\alpha_{1}}{\sigma_{P}^{2}}\left(\mathbf{R P}-\alpha_{0}\right)^{T} \Sigma_{H}^{-1}+\frac{\beta^{T} F^{T} \Sigma_{K}^{-1}}{\sigma_{T}^{2}}\right) \mathbf{T}\right]\right] \\ & \propto \exp \left[-\frac{1}{2}\left[T^{T} \Omega^{-1} T-2 \Delta^{T} \Omega \Omega^{-1} T+\Delta \Omega \Omega^{-1} \Omega \Delta^{T}\right]\right]\end{aligned}$

where $\Omega^{-1}=\frac{\alpha_{1}^{2}}{\sigma_{P}^{2}} \Sigma_{H}^{-1}+\frac{\Sigma_{K}^{-1}}{\sigma_{T}^{2}}$ and $\Delta=\frac{\alpha_{1}}{\sigma_{P}^{2}}\left(\mathbf{R P}-\alpha_{0}\right)^{T} \Sigma_{H}^{-1}+\frac{\beta^{T} F^{T} \Sigma_{K}^{-1}}{\sigma_{T}^{2}}$. Finally:

$$
\mathbf{T} \mid \mathbf{R P}, \theta \sim N_{M}\left(\Delta^{T} \Omega, \Omega\right) .
$$

Distribution of $\alpha \mid \mathbf{R P}, \mathbf{T}, \sigma_{P}^{2}, H$. For ease of calculation, assume that $\alpha$ is independent of $\mathbf{T}$ and $\sigma_{P}^{2}$ and $H$ at the prior level. Then:

$$
\begin{aligned}
{\left[\alpha \mid \mathbf{R P}, \mathbf{T}, \sigma_{P}^{2}, H\right] } & \propto \exp \left[-\frac{1}{2}\left[\alpha^{T}\left(\frac{1}{\sigma_{P}^{2}} \tilde{\mathbf{T}}^{T} \Sigma_{H}^{-1} \tilde{\mathbf{T}}+\hat{D}_{\alpha}^{-1}\right) \alpha-2\left(\frac{\mathbf{R P}^{T} \Sigma_{H}^{-1} \tilde{\mathbf{T}}}{\sigma_{P}^{2}}+\hat{\alpha}^{T} \hat{D}_{\alpha}^{-1}\right) \alpha\right]\right] \\
& \propto \exp \left[-\frac{1}{2}\left[\alpha^{T} \Omega_{\alpha}^{-1} \alpha-2 \Delta_{\alpha} \Omega_{\alpha}^{-1} \alpha+\Delta_{\alpha} \Omega_{\alpha} \Omega_{\alpha}^{-1} \Omega_{\alpha} \Delta_{\alpha}^{T}\right]\right]
\end{aligned}
$$

where $\Omega_{\alpha}^{-1}=\frac{1}{\sigma_{P}^{2}} \tilde{\mathbf{T}}^{T} \Sigma_{H}^{-1} \tilde{\mathbf{T}}+\hat{D}_{\alpha}^{-1}$ and $\Delta_{\alpha}=\frac{\mathbf{R P}^{T} \Sigma_{H}^{-1} \tilde{\mathbf{T}}}{\sigma_{P}^{2}}+\hat{\alpha}^{T} \hat{D}_{\alpha}^{-1}$. Hence:

$$
\alpha \mid \mathbf{R P}, \mathbf{T}, \sigma_{P}^{2}, H \sim N_{2}\left(\Delta_{\alpha} \Omega_{\alpha}, \Omega_{\alpha}\right) .
$$

Distribution of $\beta \mid \mathbf{T}, \sigma_{T}^{2}, K$. For ease of calculation, let us assume that $\beta$ is independent of $\sigma_{T}^{2}$ and $K$ at the prior level. Then:

$$
\begin{aligned}
{\left[\beta \mid \mathbf{T}, \sigma_{T}^{2}, K\right] } & \propto \exp \left[-\frac{1}{2}\left[\beta^{T}\left(\frac{1}{\sigma_{T}^{2}} \mathbf{F}^{T} \Sigma_{K}^{-1} \mathbf{F}+\hat{D}_{\beta}^{-1}\right) \beta-2\left(\frac{\mathbf{T}^{T} \Sigma_{K}^{-1} \mathbf{F}}{\sigma_{T}^{2}}+\hat{\beta} \hat{D}_{\beta}^{-1}\right) \beta\right]\right] \\
& \propto \exp \left[-\frac{1}{2}\left[\beta^{T} \Omega_{\beta}^{-1} \beta-2 \Delta_{\beta} \Omega_{\beta}^{-1} \beta+\Delta_{\beta} \Omega_{\beta} \Omega_{\beta}^{-1} \Omega_{\beta} \Delta_{\beta}^{T}\right]\right]
\end{aligned}
$$

where

$$
\Omega_{\beta}^{-1}=\frac{1}{\sigma_{T}^{2}} \mathbf{F}^{T} \Sigma_{K}^{-1} \mathbf{F}+\hat{D}_{\beta}^{-1}
$$


and $\Delta_{\beta}=\frac{\mathbf{T}^{T} \Sigma_{K}^{-1} \mathbf{F}}{\sigma_{T}^{2}}+\hat{\beta} \hat{D}_{\beta}^{-1}$. Therefore:

$$
\beta \mid \mathbf{T}, \sigma_{T}^{2}, K \sim N_{4}\left(\Delta_{\beta} \Omega_{\beta}, \Omega_{\beta}\right) .
$$

Distribution of $\sigma_{P}^{2} \mid \mathbf{R P}, \mathbf{T}, \alpha, H$.

$$
\begin{aligned}
{\left[\sigma_{P}^{2} \mid \mathbf{R P}, \mathbf{T}, \alpha, H\right] } & =\frac{\exp \left[-\frac{1}{2 \sigma_{P}^{2}}(\mathbf{R P}-\tilde{\mathbf{T}} \alpha)^{T} \Sigma_{H}^{-1}(\mathbf{R P}-\tilde{\mathbf{T}} \alpha)\right]}{\sqrt{(2 \pi)^{M}\left|\Sigma_{H} \sigma_{P}^{2}\right|}}\left(\sigma_{P}^{2}\right)^{-q_{1}-1} \exp \left[-\frac{r_{1}}{\sigma_{P}^{2}}\right] \\
& \propto\left(\sigma_{P}^{2}\right)^{-\left(q_{1}^{\prime}+1\right)} \exp \left[-\frac{r_{1}^{\prime}}{\sigma_{P}^{2}}\right]
\end{aligned}
$$

where $q_{1}^{\prime}=q_{1}+\frac{M}{2}$ and $r_{1}^{\prime}=r_{1}+\frac{1}{2}(\mathbf{R P}-\tilde{\mathbf{T}} \alpha)^{T} \Sigma_{H}^{-1}(\mathbf{R P}-\tilde{\mathbf{T}} \alpha)$. Then:

$$
\sigma_{P}^{2} \mid \mathbf{R P}, \mathbf{T}, \beta, H \sim I G\left(q_{1}^{\prime}, r_{1}^{\prime}\right) .
$$

Distribution of $\sigma_{T}^{2} \mid \mathbf{T}, \beta, K$.

$$
\begin{aligned}
{\left[\sigma_{T}^{2} \mid \mathbf{T}, \beta, H\right] } & =\frac{\exp \left[-\frac{1}{2 \sigma_{T}^{2}}(\mathbf{T}-\mathbf{F} \beta)^{T} \Sigma_{K}^{-1}(\mathbf{T}-\mathbf{F} \beta)\right]}{\sqrt{(2 \pi)^{M}\left|\Sigma_{K} \sigma_{T}^{2}\right|}} \cdot\left(\sigma_{T}^{2}\right)^{-q_{2}-1} \exp \left[-\frac{r_{2}}{\sigma_{T}^{2}}\right] \\
(\text { A.5 }) & \propto\left(\sigma_{T}^{2}\right)^{-\left(q_{2}^{\prime}+1\right)} \exp \left[-\frac{r_{2}^{\prime}}{\sigma_{T}^{2}}\right]
\end{aligned}
$$

where $q_{2}^{\prime}=q_{2}+\frac{M}{2}$ and $r_{2}^{\prime}=r_{2}+\frac{1}{2}(T-F \beta)^{T} \Sigma_{K}^{-1}(T-F \beta)$. Therefore:

$$
\sigma_{T}^{2} \mid \mathbf{T}, \beta, K \sim I G\left(q_{2}^{\prime}, r_{2}^{\prime}\right)
$$

Distribution of $\mathbf{T}_{1} \mid \mathbf{T}_{2}, \theta$. Let $\Delta^{T} \Omega=\left[\mu_{1 T}, \mu_{2 T}\right]$ and $\Omega=\left[\begin{array}{ll}\Omega_{11} & \Omega_{12} \\ \Omega_{21} & \Omega_{22}\end{array}\right]$ the corresponding decomposition of $\Omega$ and $\Delta^{T} \Omega$ according to the dimensions of $\mathbf{T}=\left[\mathbf{T}_{1}, \mathbf{T}_{2}\right]$. If $\tilde{\mathbf{T}}_{2}$ is a realization (or observations) of the random variable $\mathbf{T}_{2}$ then:

$$
\mathbf{T}_{1} \mid \mathbf{T}_{2}, \theta \sim N(\bar{\mu}, \bar{\Omega})
$$

where $\bar{\mu}=\mu_{1 T}+\Omega_{12} \Omega_{22}^{-1}\left(\tilde{\mathbf{T}}_{2}-\mu_{2 T}\right)$ and $\bar{\Omega}=\Omega_{11}-\Omega_{12} \Omega_{22}^{-1} \Omega_{21}$. 
Distribution of $H \mid \mathbf{R P}, \mathbf{T}, \alpha, \sigma_{P}^{2}$. After similar calculations as the previous cases, the posterior distribution of $H$ turns out to be proportional to:

$\left[H \mid \mathbf{R P}, \mathbf{T}, \alpha, \sigma_{P}^{2}\right] \propto\left[\operatorname{det}\left(\Sigma_{H}\right)\right]^{-1 / 2} \exp \left[-\frac{1}{2 \sigma_{P}^{2}}(\mathbf{R P}-\tilde{\mathbf{T}} \alpha)^{T} \Sigma_{H}^{-1}(\mathbf{R P}-\tilde{\mathbf{T}} \alpha)\right]$

where we are assuming that the prior of $H$ does not depend on $\alpha$ and $\sigma_{P}^{2}$. It is inmediate that the posterior does not have a known distribution, then a Metropolis-Hastings is preferable for the sampling process. We assume the proposal distribution at repetition $i(1<i \leq R, R$ : total number of repetitions of the $\mathrm{MCMC})$ :

$$
p\left(H \mid H^{(i-1)}\right)=T N\left(H^{(i-1)}, S_{a}(i), 0, K^{(i)}\right)
$$

where $H^{(i)}$ is the i-th sample of $H, K^{(i)}$ is the i-th sample of $K, S_{a}(i)=\left(D_{0}-\right.$ $\left.D_{1}\right) \cdot A R_{i}+D_{1}\left(A R_{i}\right.$ is the acceptance ratio of $H$ at time $i$ and $\left.D_{0}>D_{1}>0\right)$ and $T N(\mu, \sigma, a, b)$ stands for a Truncated Normal distribution with mean $\mu$, standard deviation $\sigma$ and lower and upper limit $a$ and $b$ respectively.

Distribution of $K \mid \mathbf{T}, \beta, \sigma_{T}^{2}$. After some calculations the posterior distribution of $K$ is proportional to:

$$
\left[K \mid \mathbf{T}, \beta, \sigma_{T}^{2}\right] \propto\left[\operatorname{det}\left(\Sigma_{K}\right)\right]^{-1 / 2} \exp \left[-\frac{1}{2 \sigma_{T}^{2}}(\mathbf{T}-\mathbf{F} \beta)^{T} \Sigma_{K}^{-1}(\mathbf{T}-\mathbf{F} \beta)\right]
$$

The Metropolis-Hastings algorithm is employed here using the proposal distribution:

$$
p\left(K \mid K^{(i-1)}\right)=T N\left(K^{(i-1)}, S_{a}(i), 0,1\right)
$$

where as before $1<i \leq R$ and $K^{(i)}$ is the $\mathrm{i}$-th member of the simulated Metropolis-Hastings sample. $S_{a}(i)$ is given as the previous section, but it depends now on the acceptance ratio of the current sample of $K$.

\section{REFERENCES}

Baillie, R. T., Bollerslev, T. and Mikkelsen, H. O. (1996). Fractionally integrated generalized autoregressive conditional heteroskedasticity. Journal of Econometrics $\mathbf{7 4}$ $3-30$.

Beran, J. (1992). A Goodness-of-Fit Test for Time Series with Long Range Dependence. Journal of the Royal Statistical Society. Series B (Methodological) $\mathbf{5 4 .}$

Beran, J. (1994). Statistics for long-memory processes. Monographs on Statistics and Applied Probability, 61. Chapman \& Hall.

Biagini, F., Hu, Y., Øksendal, B. and Zhang, T. (2010). Stochastic Calculus for Fractional Brownian Motion and Applications (Probability and Its Applications), 1st ed. Springer. 
Brouste, A. and Iacus, S. M. (2012). Parameter estimation for the discretely observed fractional OrnsteinUhlenbeck process and the Yuima R package. Computational Statistics 28 1529-1547.

Chronopoulou, A., Viens, F. G. and Tudor, C. A. (2009). Variations and Hurst index estimation for a Rosenblatt process using longer filters. Electronic Journal of Statistics 3 1393-1435.

Coeurjolly, J.-F. (2001). Estimating the Parameters of a Fractional Brownian Motion by Discrete Variations of its Sample Paths. Statistical Inference for Stochastic Processes 4 199-227.

Davies, R. B. and Harte, D. S. (1987). Tests for Hurst Effect. Biometrika 74 95-101.

Gneiting, T., Balabdaoui, F. and Raftery, A. E. (2007). Probabilistic forecasts, calibration and sharpness. Journal of the Royal Statistical Society: Series B (Statistical Methodology) 69 243-268.

Gneiting, T. and Raftery, A. E. (2007). Strictly Proper Scoring Rules, Prediction, and Estimation. Journal of the American Statistical Association 102 359-378.

Granger, C. W. J. and Joyeux, R. (1980). An Introduction to Long-Memory Time Series Models and Fractional Differencing. Journal of Time Series Analysis 1 15-29.

Gschlössl, S. and CzAdo, C. (2007). Spatial modelling of claim frequency and claim size in non-life insurance. Scandinavian Actuarial Journal 2007 202-225.

IstAs, J. and LANG, G. (1997). Quadratic variations and estimation of the local Hölder index of a Gaussian process. Annales de l'Institut Henri Poincaré (B) Probability and Statistics 33 407-436.

Kleptsyna, M. L. and Le Breton, A. (2002). Statistical Analysis of the Fractional Ornstein-Uhlenbeck Type Process. Statistical Inference for Stochastic Processes 5 229248.

Nualart, D. (2010). The Malliavin Calculus and Related Topics (Probability and Its Applications), Second ed. Springer.

Percival, D. B. and Walden, A. T. (1993). Spectral Analysis for Physical Applications, First ed. Cambridge University Press.

Robinson, P. M. (1995). Gaussian Semiparametric Estimation of Long Range Dependence. The Annals of Statistics 23 1630-1661.

Samorodnitsky, G. and TAqQu, M. (1994). Stable Non-Gaussian Random Processes: Stochastic Models with Infinite Variance, First ed. Stochastic modeling. Chapman and Hall/CRC.

Thomson, D. J. (1982). Spectrum estimation and harmonic analysis. Proceedings of the IEEE 70 1055-1096.

Tudor, C. A. and Viens, F. G. (2007). Statistical Aspects of the Fractional Stochastic Calculus. The Annals of Statistics 35 1183-1212.

Walden, A. T., McCoy, E. J. and Percival, D. B. (1995). The Effective Bandwidth of a Multitaper Spectral Estimator. Biometrika 82 201-214. 


\section{ONLINE SUPPLEMENT B: ADDITIONAL PLOTS AND TABLES}

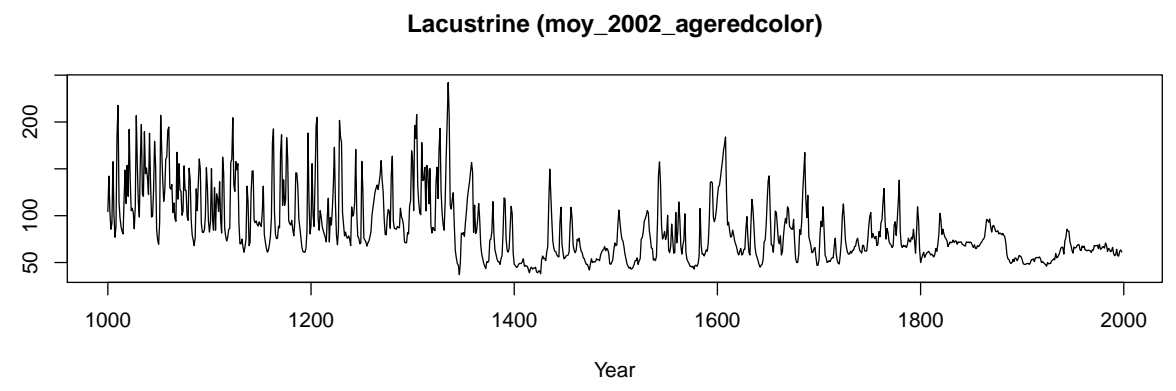

Speleothem (lee_thorpe_2001_c13)

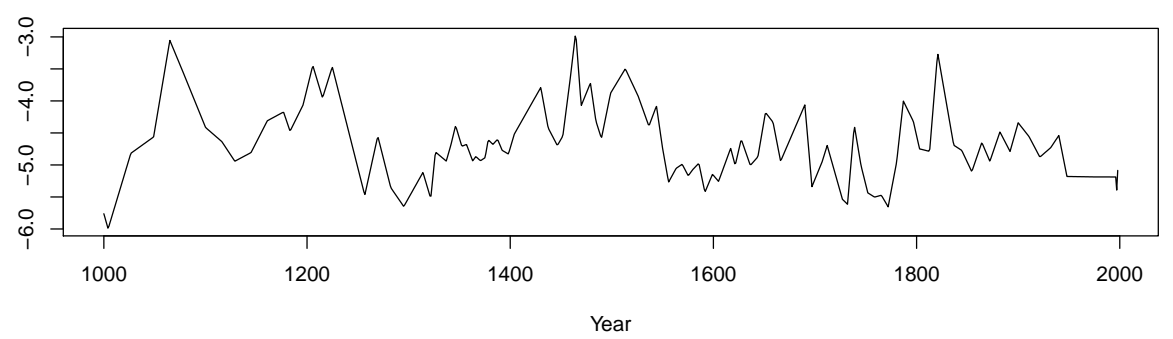

Fig B.1: Two examples of individual proxy series.

TABLE B. 1

Proxies not included in $R P_{t}$

\begin{tabular}{l|l|l} 
Name & Type & Country \\
\hline burns_2003_socotrad13c & Speleothem & Yemen \\
burns_2003_socotrad18o & Speleothem & Yemen \\
burns_nicoya_d18o & Speleothem & Costa Rica \\
curtis_1996_d13cpyro & Lacustrine & Mexico \\
curtis_1996_d18o & Lacustrine & Mexico \\
dongge & Speleothem & China \\
fisher_1994_agassiz & Ice core & Canada \\
ge_2002_10yeartemp & Other & China \\
lee_thorpe_2001_c13 & Speleothem & South Africa \\
lee_thorpe_2001_o18 & Speleothem & South Africa \\
moy_2002_ageredcolor & Lacustrine & Ecuador \\
tiljander_2003_lightsum & Lacustrine & Finland \\
tiljander_2003_thicknessmm & Lacustrine & Finland \\
\hline
\end{tabular}


TABle B. 2

Least Squares estimates of model (3.1). HAD and CRU temperature datasets.

\begin{tabular}{l|l|r|r}
\hline Proxy & Type & HAD & CRU \\
\hline ak046 & Tree Ring & 0.034 & 0.021 \\
\hline arge091 & Tree Ring & 0.008 & 0.021 \\
\hline az510 & Tree Ring & 0.015 & 0.045 \\
\hline baker_2002_su.96.7 & Speleothem & 0.035 & -0.003 \\
\hline ca528 & Tree Ring & 0.002 & -0.023 \\
\hline ca529 & Tree Ring & 0.019 & 0.026 \\
\hline ca630 & Tree Ring & 0.030 & 0.037 \\
\hline co522 & Tree Ring & -0.024 & -0.020 \\
\hline fisher_1996_cgreenland & Tree Ring & -0.003 & -0.028 \\
\hline gisp2o18 & Ice core & -0.007 & -0.012 \\
\hline mongolia.darrigo & Ice core & 0.014 & 0.030 \\
\hline mt110 & Other & 0.038 & 0.058 \\
\hline norw010 & Tree Ring & -0.013 & -0.009 \\
\hline nv512 & Tree Ring & 0.022 & 0.030 \\
\hline nv513 & Tree Ring & 0.033 & 0.015 \\
\hline nv518 & Tree Ring & -0.008 & 0.004 \\
\hline nv519 & Tree Ring & 0.025 & 0.043 \\
\hline orokonztr & Tree Ring & -0.014 & -0.020 \\
\hline pola006 & Other & 0.014 & 0.014 \\
\hline qian_2003_yriver & Tree Ring & 0.001 & 0.010 \\
\hline tasmania_recon_orig & Other & -0.015 & -0.029 \\
\hline thompson_1992_quelccao18 & Other & 0.013 & 0.027 \\
\hline tiljander_2003_darksum & Ice core & 0.024 & 0.017 \\
\hline tornetrask & Lacustrine & 0.036 & 0.038 \\
\hline \hline$R^{2}$ & Tree Ring & 0.020 & 0.032 \\
\hline & & $77.48 \%$ & $58.25 \%$ \\
\hline & & & \\
\hline
\end{tabular}

TABle B.3

Relative magnitude of each LS coefficient, classified by country and proxy type (HAD)

\begin{tabular}{|c|c|c|c|c|c|c|}
\hline & Tree Ring & Ice core & Lacustrine & Speleothem & Other & Total \\
\hline Argentina & $1.77 \%$ & & & & & $1.77 \%$ \\
\hline China & & & & & $3.18 \%$ & $3.18 \%$ \\
\hline Finland & & & $7.64 \%$ & & & $7.64 \%$ \\
\hline Greenland & & $4.47 \%$ & & & & $4.47 \%$ \\
\hline Mongolia & & & & & $8.22 \%$ & $8.22 \%$ \\
\hline New Zealand & & & & & $3.06 \%$ & $3.06 \%$ \\
\hline Norway & $4.76 \%$ & & & & & $4.76 \%$ \\
\hline Peru & & $5.05 \%$ & & & & $5.05 \%$ \\
\hline Poland & $0.16 \%$ & & & & & $0.16 \%$ \\
\hline Scotland & & & & $7.54 \%$ & & $7.54 \%$ \\
\hline Sweden & $4.27 \%$ & & & & & $4.27 \%$ \\
\hline Tasmania & & & & & $2.88 \%$ & $2.88 \%$ \\
\hline USA & $46.99 \%$ & & & & & $46.99 \%$ \\
\hline Total & $57.96 \%$ & $9.52 \%$ & $7.64 \%$ & $7.54 \%$ & $17.34 \%$ & $100.00 \%$ \\
\hline
\end{tabular}


TABle B. 4

Relative magnitude of each LS coefficient, classified by country and proxy type (CRU)

\begin{tabular}{|c|c|c|c|c|c|c|}
\hline & Tree Ring & Ice core & Lacustrine & Speleothem & Other & Total \\
\hline Argentina & $3.50 \%$ & & & & & $3.50 \%$ \\
\hline China & & & & & $4.70 \%$ & $4.70 \%$ \\
\hline Finland & & & $6.28 \%$ & & & $6.28 \%$ \\
\hline Greenland & & $6.84 \%$ & & & & $6.84 \%$ \\
\hline Mongolia & & & & & $9.46 \%$ & $9.46 \%$ \\
\hline New Zealand & & & & & $2.27 \%$ & $2.27 \%$ \\
\hline Norway & $4.98 \%$ & & & & & $4.98 \%$ \\
\hline Peru & & $2.79 \%$ & & & & $2.79 \%$ \\
\hline Poland & $1.63 \%$ & & & & & $1.63 \%$ \\
\hline Scotland & & & & $0.46 \%$ & & $0.46 \%$ \\
\hline Sweden & $5.27 \%$ & & & & & $5.27 \%$ \\
\hline Tasmania & & & & & $4.34 \%$ & $4.34 \%$ \\
\hline USA & $47.47 \%$ & & & & & $47.47 \%$ \\
\hline Total & $62.87 \%$ & $9.63 \%$ & $6.28 \%$ & $0.46 \%$ & $20.76 \%$ & $100.00 \%$ \\
\hline
\end{tabular}



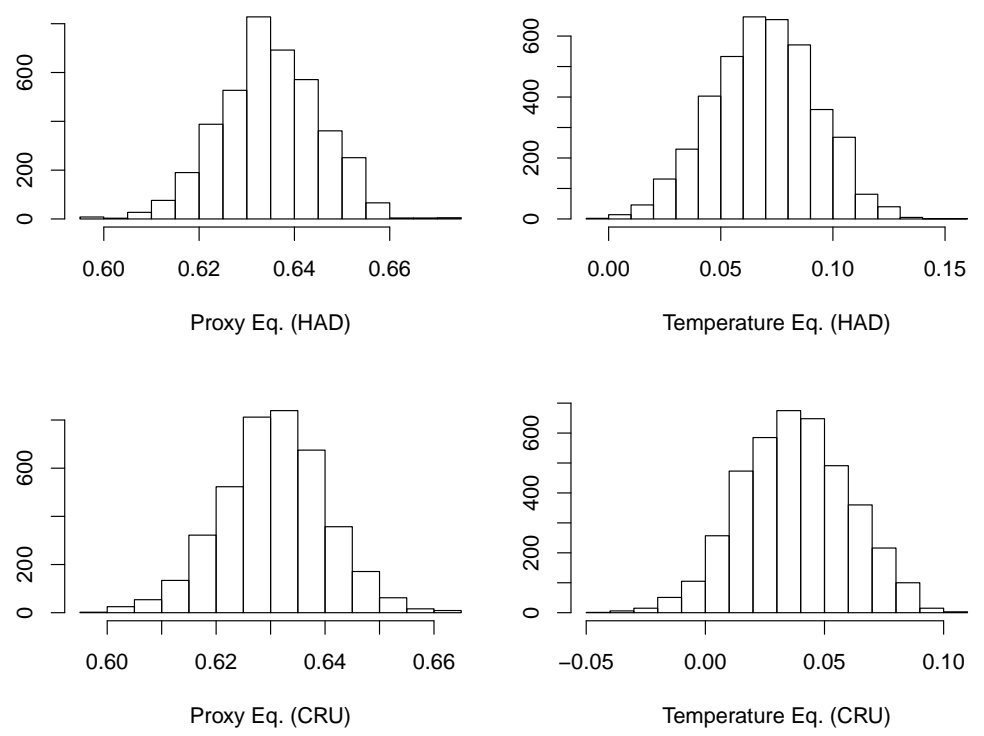

Fig B.2: Bayesian Estimation of Error parameters. Scenario B.
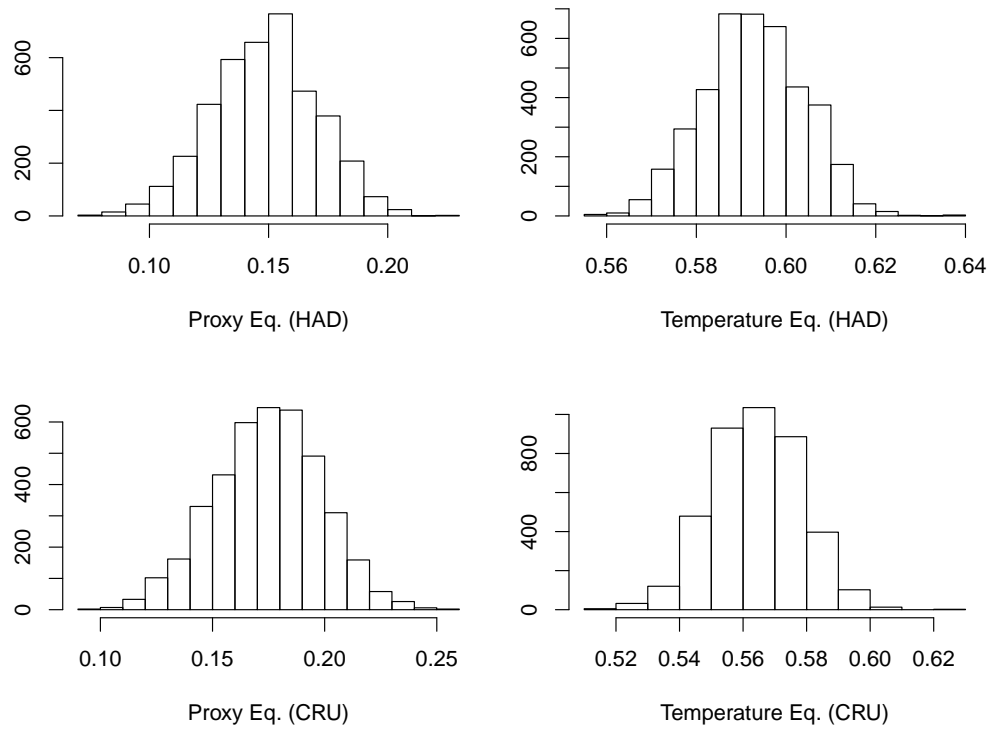

Fig B.3: Bayesian Estimation of Error parameters. Scenario C. 

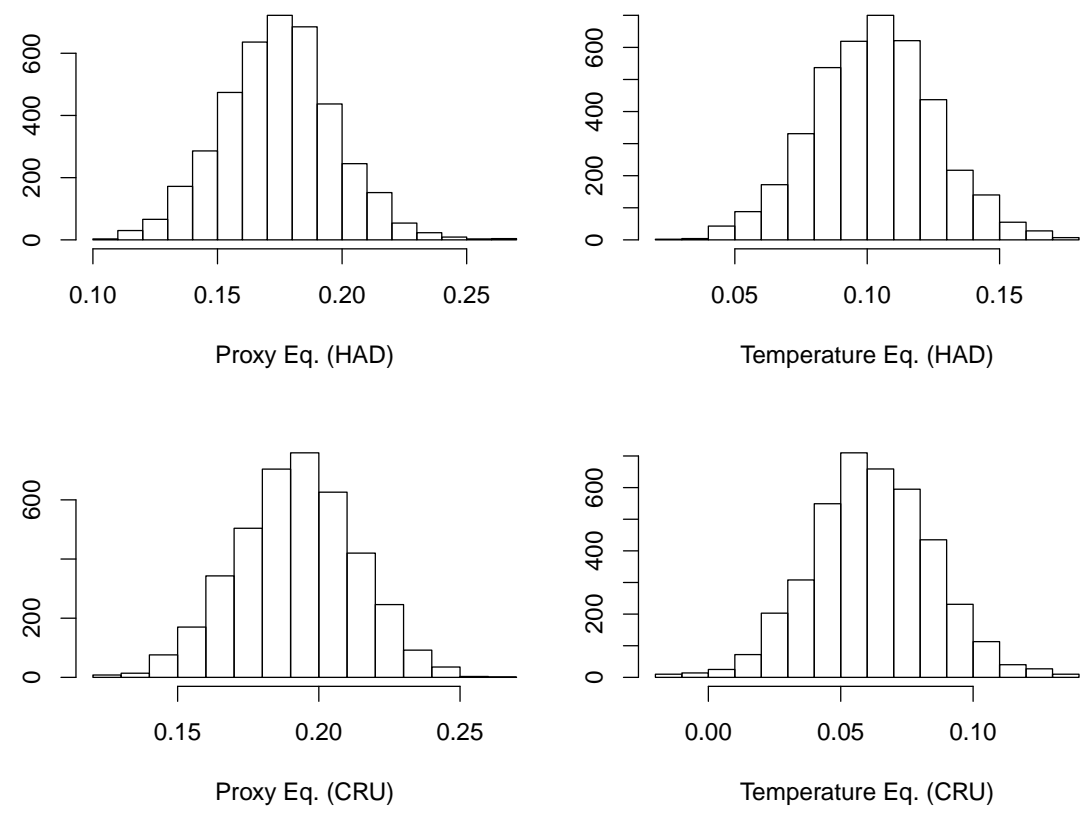

Fig B.4: Bayesian Estimation of Error parameters. Scenario D. 

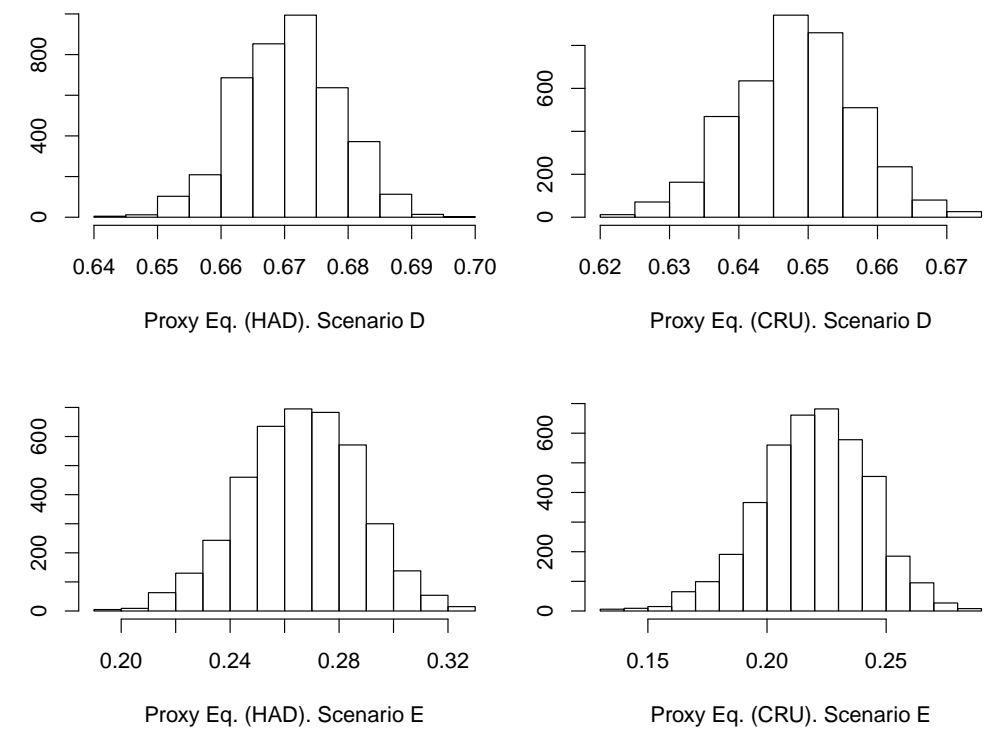

Fig B.5: Bayesian Estimation of Error parameters in the Proxy equation. Scenarios F and G. 

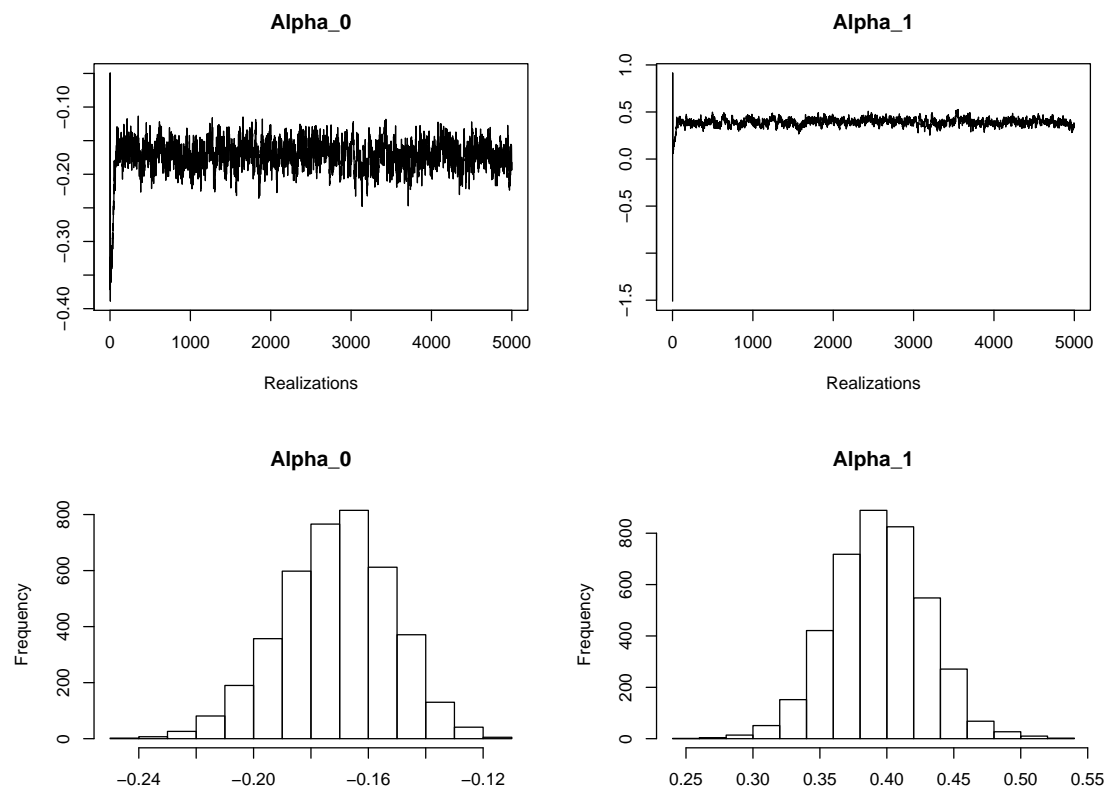

Fig B.6: Bayesian Estimation of $\alpha$. (HAD, Scenario A)
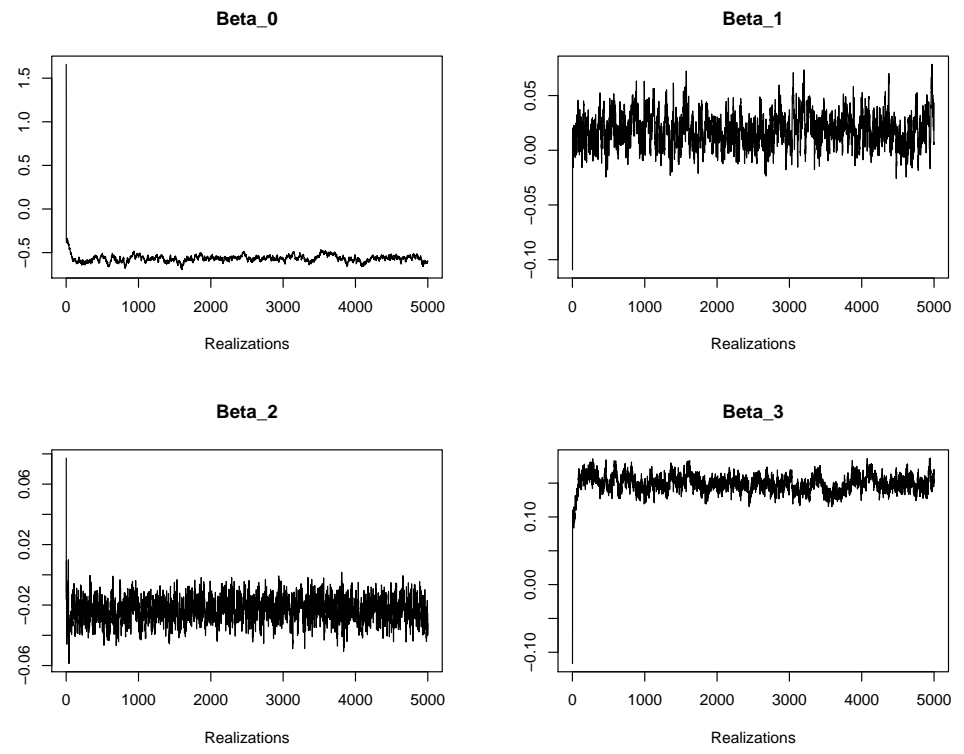

Fig B.7: Bayesian Estimation of $\beta$. (Realizations) (HAD, Scenario A) 

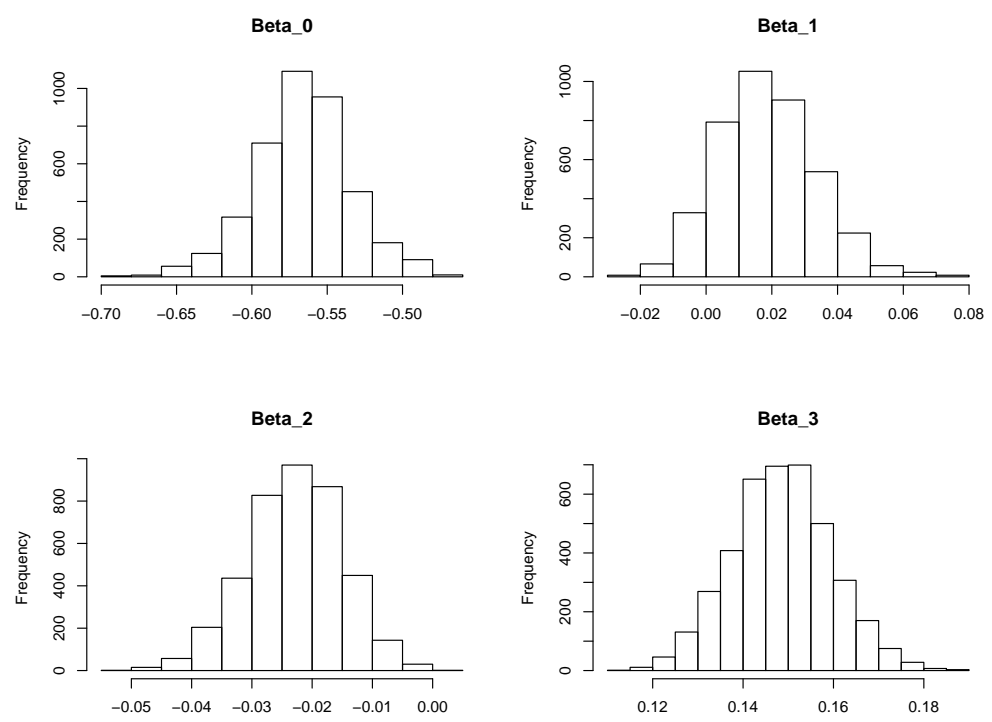

Fig B.8: Bayesian Estimation of $\beta$. (Histograms) (HAD, Scenario A)
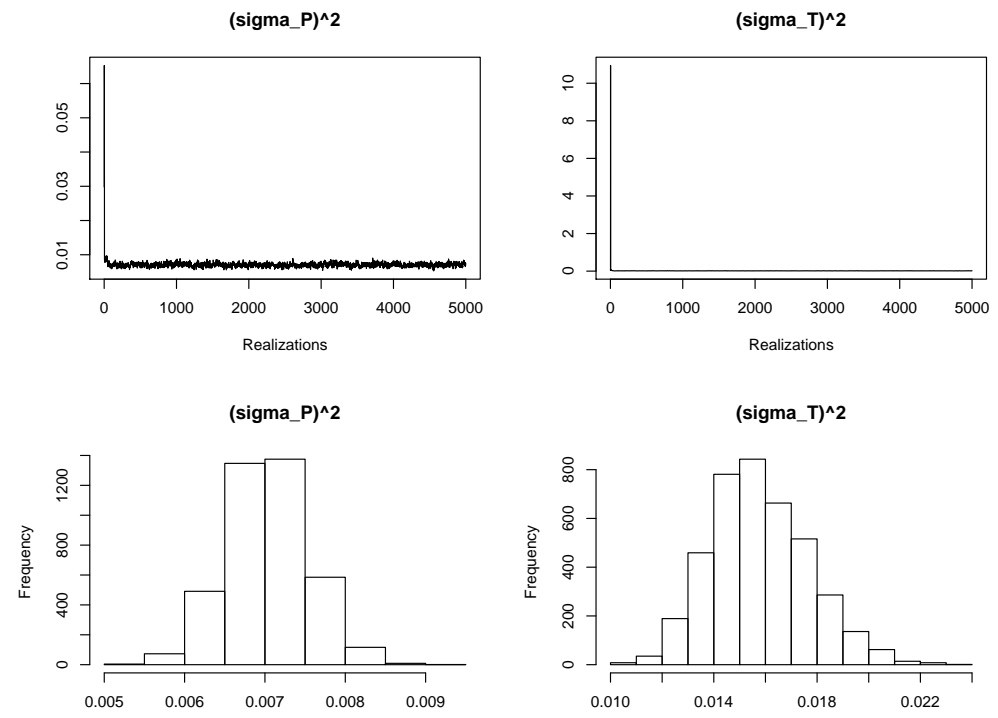

Fig B.9: Bayesian Estimation of $\sigma_{P}^{2}$ and $\sigma_{T}^{2}$. (HAD, Scenario A) 

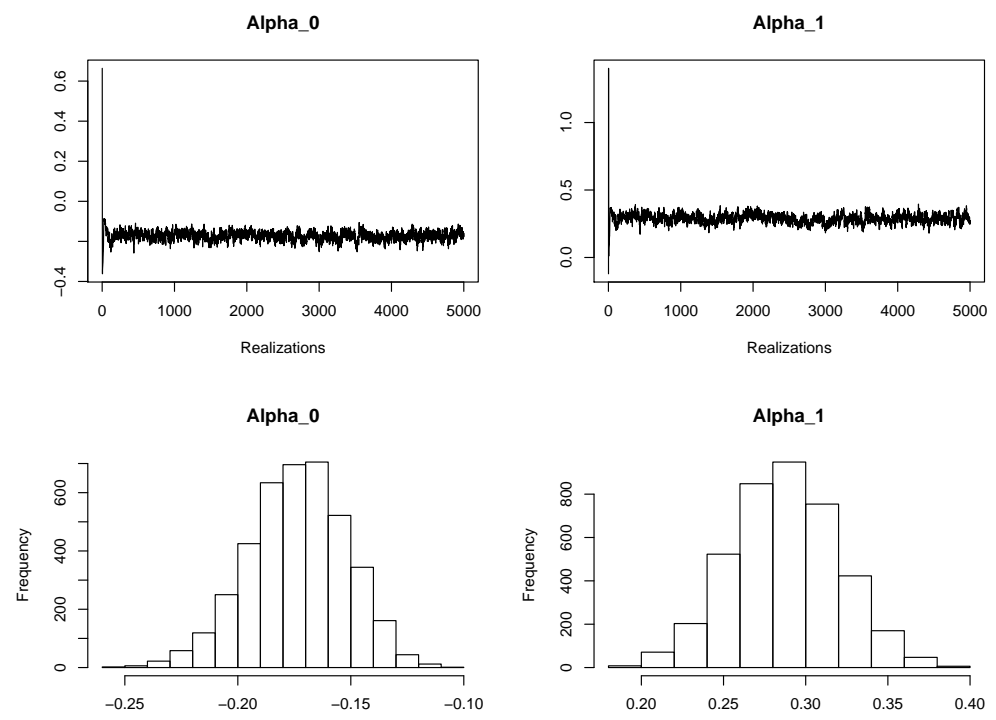

Fig B.10: Bayesian Estimation of $\alpha$. (CRU, Scenario A)
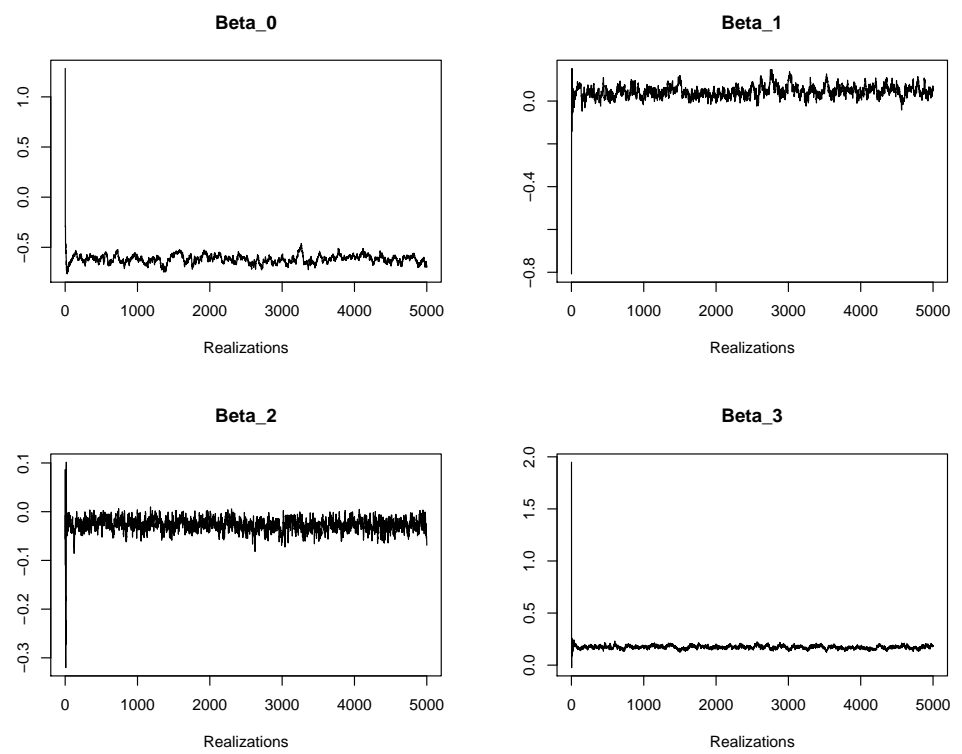

Fig B.11: Bayesian Estimation of $\beta$. (Realizations) (CRU, Scenario A) 

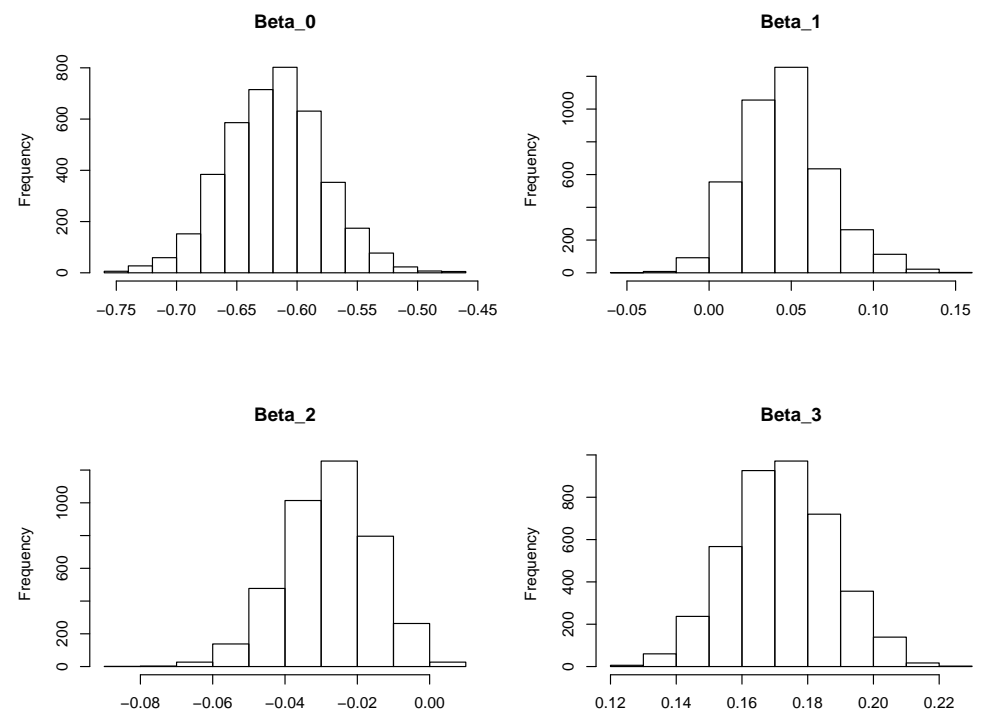

Fig B.12: Bayesian Estimation of $\beta$. (Histograms) (CRU, Scenario A)
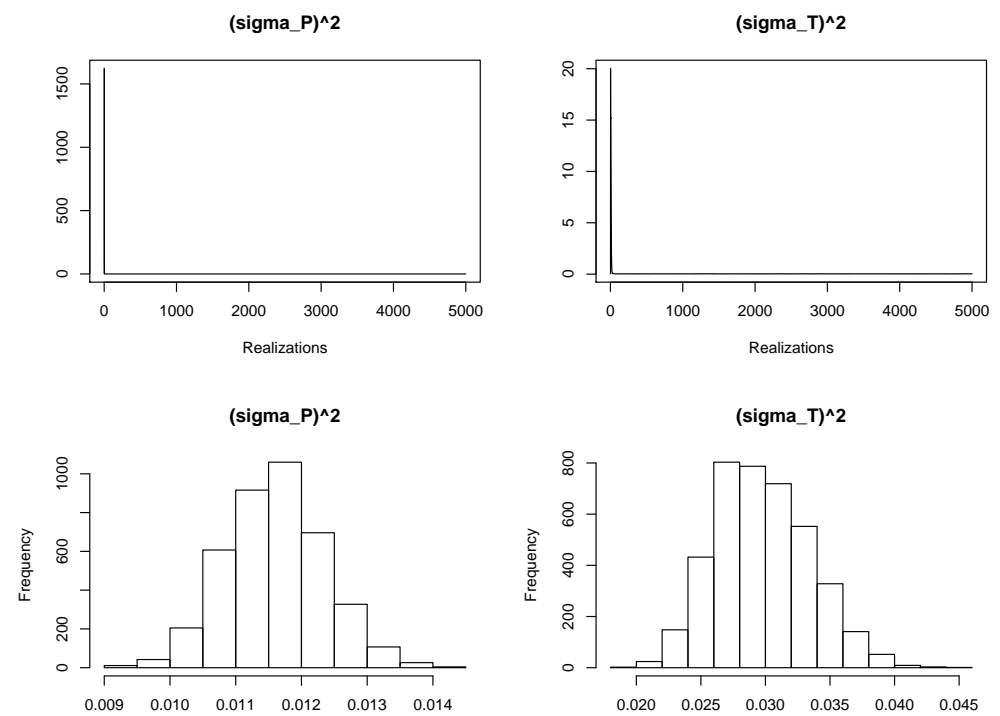

Fig B.13: Bayesian Estimation of $\sigma_{P}^{2}$ and $\sigma_{T}^{2}$. (CRU, Scenario A) 


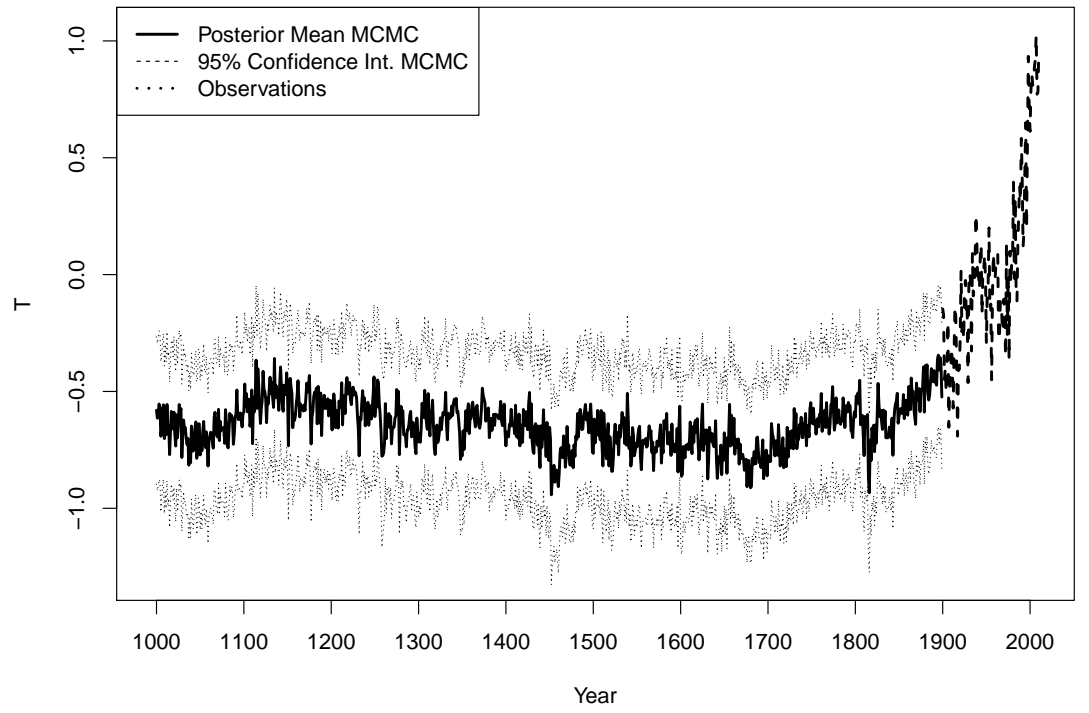

Fig B.14: Temperature reconstruction (1000-1899) using the CRU dataset, Scenario B. 

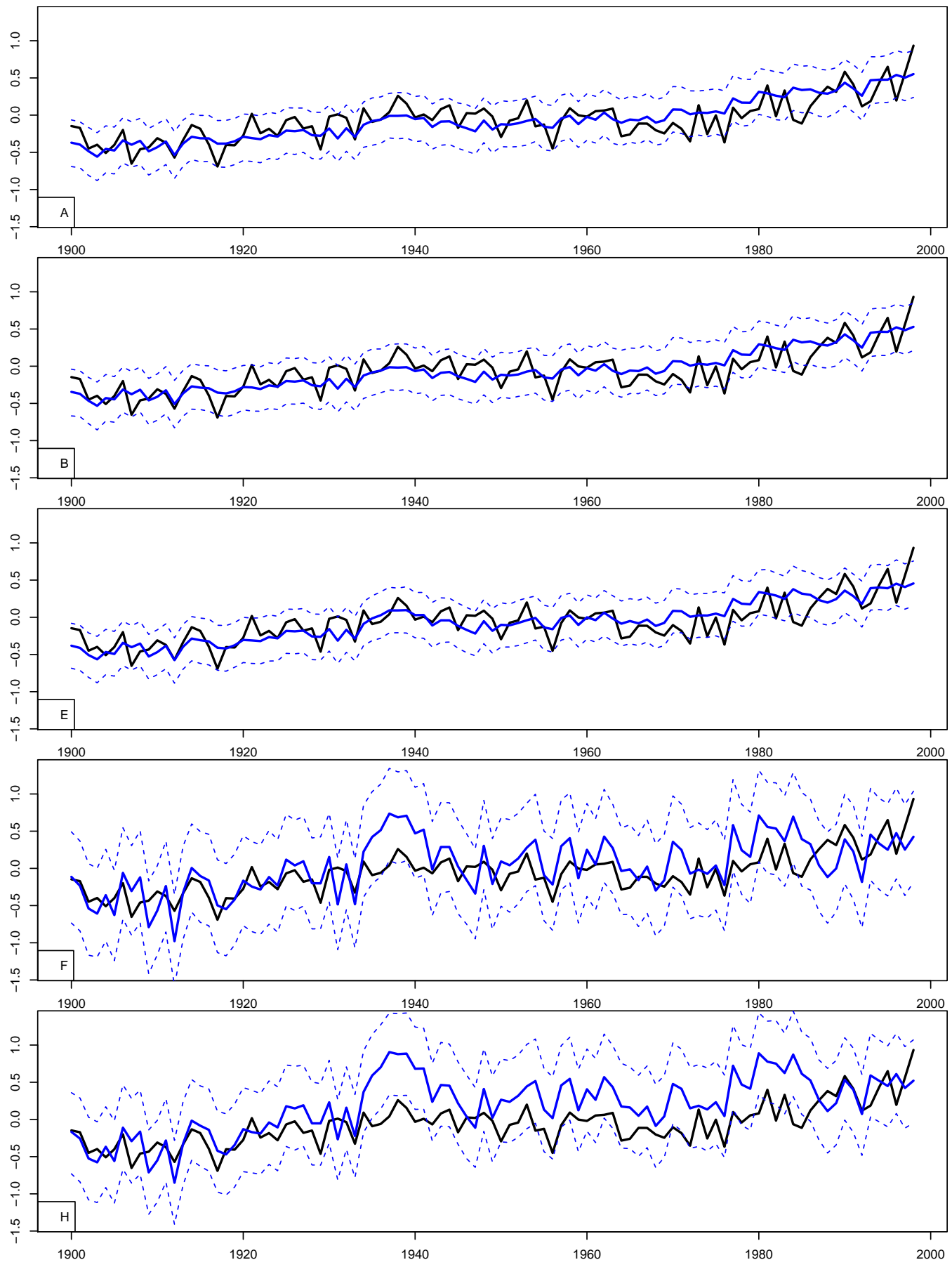

Fig B.15: Temperature reconstruction (1900-1998) using the CRU dataset under Scenarios A, B, E, F and H. Black: Observations; Purple: 95\% credible intervals MCMC; Dark Purple line: posterior mean MCMC. 

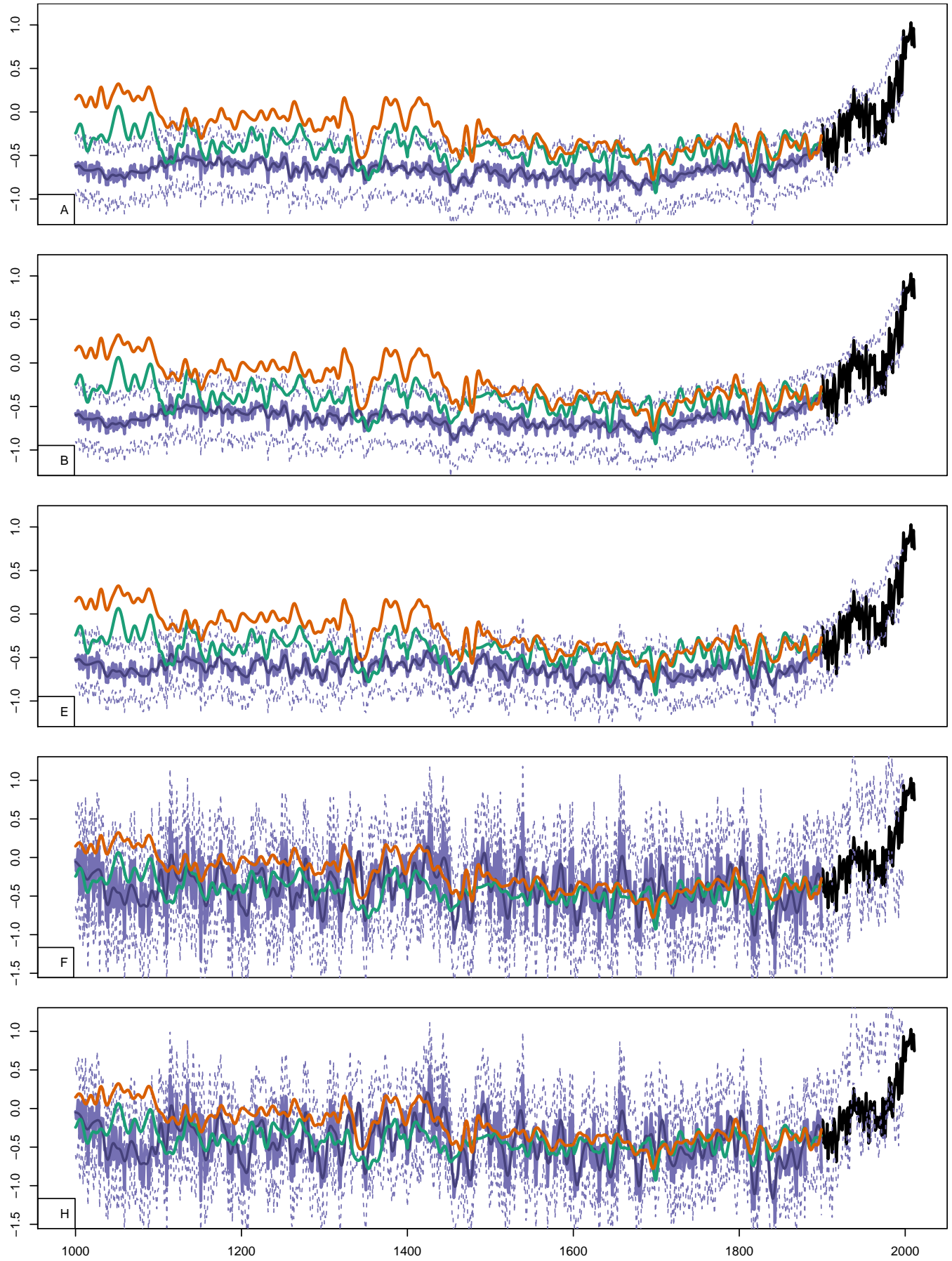

Fig B.16: Comparison of Scenarios A, B, E, F, H with previous reconstructions (CRU). Black: Observations, Purple: MCMC posterior mean with 95\% credible intervals, Gold: EIV (Mann et al., 2008), Green: CPS (Mann et al., 2008). Dark Purple line: mean of smoothed posteriors. 

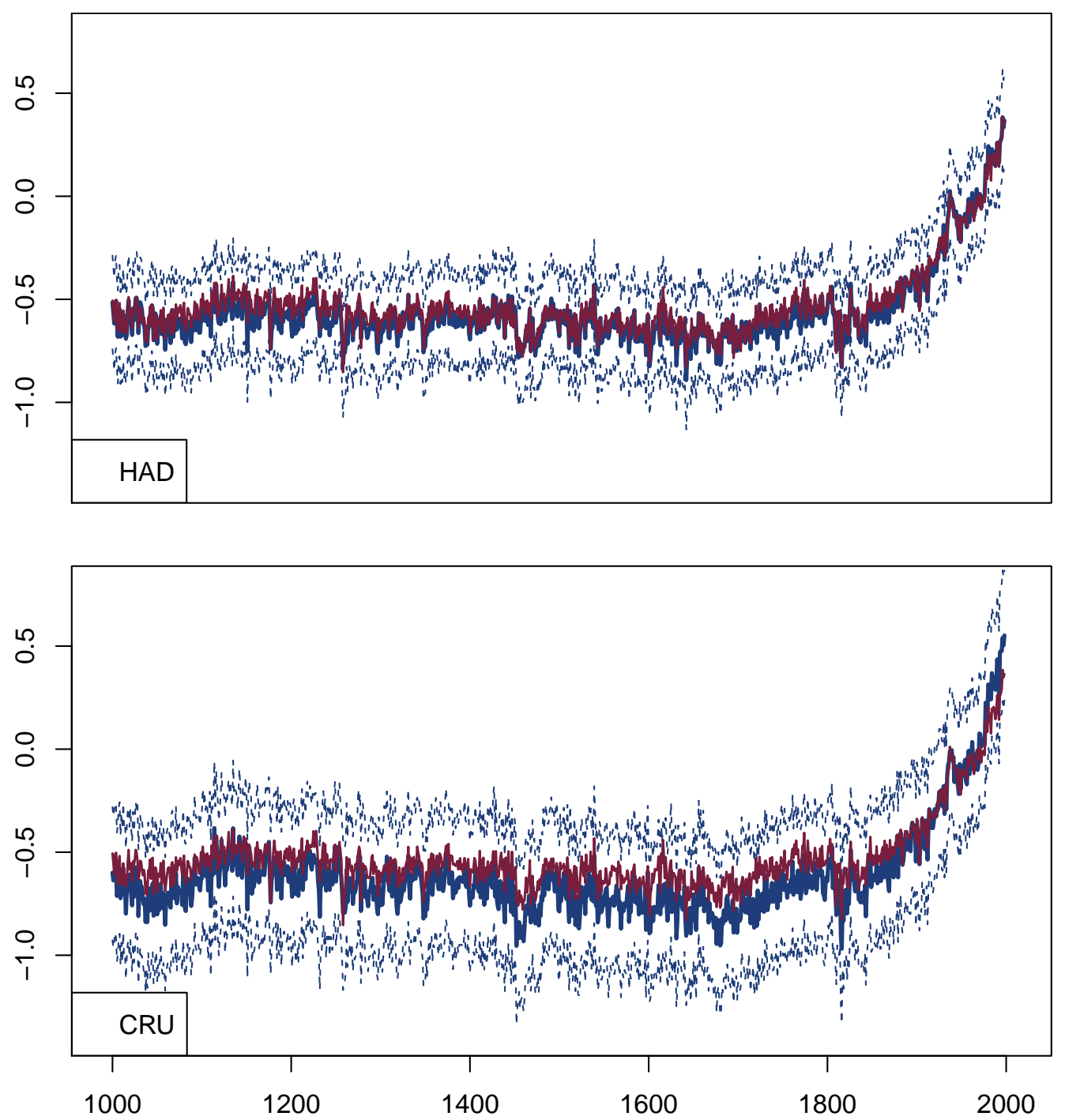

Fig B.17: Comparison of HAD and CRU reconstructions with and without the single lacustrine series in $R P_{t}$ (Scenario A). Blue line: posterior mean of the original MCMC reconstruction, blue dotted line: $95 \%$ prediction intervals of the original MCMC reconstruction, red line: posterior mean of the MCMC reconstruction without the lacustrine series. 